\title{
Parametric analysis of the nonlinear behavior of rotating structures
}

\author{
Lihan XIE \\ Université de Lyon, CNRS, INSA-Lyon, LaMCoS / \\ CEA-Saclay, DEN,DANS,DM2S,SEMT,DYN, \\ Email: lihan.xie@insa-lyon.fr \\ Sébastien Baguet* \\ Université de Lyon, CNRS, INSA-Lyon, LaMCoS \\ UMR5259, F-69621, France \\ Email: sebastien.baguet@insa-lyon.fr \\ Benoit Prabel \\ CEA-Saclay, DEN,DANS,DM2S,SEMT,DYN, \\ F-91191, Gif-sur-Yvette, France \\ Email: benoit.prabel@cea.fr \\ Régis Dufour \\ Université de Lyon, CNRS, INSA-Lyon, LaMCoS \\ UMR5259, F-69621, France \\ Email: regis.dufour@insa-lyon.fr
}

In nonlinear rotordynamics, techniques can take advantage of the periodic steady state behavior to predict quickly and accurately the mass unbalance response to a series of parameters, especially with the presence of certain nonlinearities which leads to nonlinear dynamics and complicated responses. The method proposed here calculates the response curve by combining Harmonic Balance Method (HBM), Alternating Frequency-Time (AFT) method and continuation. The singular points where a stability change often arises are detected with the sign change of the Jacobian determinant and then located through a penalty method that increases the solving equation system by a completing constraint. Tracking these points, which provides an efficient way to analyze parametrically the nonlinear behavior of a system, can be fulfilled, once again, by the continuation technique.

\section{INTRODUCTION}

Generally speaking, the rotating systems utilized in the energy production have a small rotor-stator gap, are able to run during long periods, and are mounted on hydrodynamic

*Address all correspondence for other issues to this author. bearings. Rotor-stator interactions in case of blade loss, crack propagation due to fatigue, and a variable stiffness due to the nonlinear restoring forces of the bearings can make the rotordynamics nonlinear and the responses complicated: significant amplitude and frequency shifts are introduced, suband super-harmonics appear, and hysteresis occurs. It is of great importance to understand, predict and control this complicated dynamics. This is commonly achieved by means of numerical simulations due to its efficiency, low cost, repeatability, operability, etc. Reaseachers have investigated numerically the dynamic of systems with various nonlinear properties, such as rotor-stator contact [1] [2] [3], crack breathing [4] [5], hydrodynamic bearings [6] [7], on board rotor mounted on hydrodynamic bearings [8], etc.

At the design stage of nonlinear mechanical systems, a particular attention must be paid to the influence of parameters in order to reach the more efficiently as possible the configuration which leads to optimal runs. Moreover, appropriate parameters choices can avoid dangerous resonance phenomena by moving the resonance frequencies out of the operating frequency range or by decreasing the resonance amplitudes to acceptable levels. Therefore, direct paramet- 
ric analysis which quickly assesses the effect of parameters on the dynamic behavior is necessary for numerical investigation of nonlinear systems.

In vibration analysis, first of all, the resonance levels and frequencies are of primary interests in the majority of investigations of forced response. Many works aim at finding the peak vibration amplitudes in nonlinear dynamical systems. Petrov [9] [10] applies the multiharmonic balance method to compute the worst vibration cases of bladed disks with friction contact interfaces. In [11], Liao combines the shooting method and Floquet theory along with a Global Search algorithm to determine the resonant peak of nonlinear systems. Similarly, Balaram et al [12] have developed a normminimizing strategy in the coupling of continuation and $\mathrm{Ge}$ netic Algorithm to perform an optimal design.

Then, determining the local stability of a periodic solution is particularly interesting in an engineering context since only stable solutions are experimentally encountered [13]. Moreover, a change in the stability can lead to significant, qualitative, and possibly dramatic changes in the system response. Efforts have been previously made by researchers for accurate detection of the singular points which include the limit (turning) points and bifurcation points. Since the tangent stiffness matrix becomes singular at these points, most of the proposed methods use this characteristic as the critical constraint which is added to the governing equations to form a so-called augmented system defining singular points [14] [15] [16]. Filippa proposes regularization penalty of increased system to avoid the singularity of the augmented system when approaching a bifurcation point [17].

This work presents a rapid systematic method for the direct parametric analysis of the targeted vibration and the change of stability of nonlinear systems. First, the nonlinear dynamic analysis is performed in the frequency domain owing to the high computational efficiency of harmonic balance method (HBM) [1]. With the help of continuation method, all dynamic equilibrium solutions of nonlinear systems are determined. Then, for stability analysis, the Floquet theory is employed [18] [19] [20]. The determinant of the Jacobian matrix is monitored because it helps to pick out the bifurcation points from limit points [17]. The singular points are then located by adding a new constraint equation to the solving system.

Next, the equation of forced response levels and bifurcation points can be calculated directly as a function of nonlinear parameters or excitation level thanks to the added constraint function and, once again, the continuation method.

\section{EQUILIBRIUM PATH}

The equation of motion for the forced vibrations of a structure with nonlinear characteristic takes the form:

$$
M \ddot{\mathbf{x}}(t)+C \dot{\mathbf{x}}(t)+\boldsymbol{K} \mathbf{x}(t)+\mathbf{f}_{n l}(\mathbf{x}, \dot{\mathbf{x}})=\mathbf{p}(\omega, t)
$$

where $\mathbf{x}(t)$ is a vector of displacements for all $n$ degrees of freedom (DOF), $\boldsymbol{M}, \boldsymbol{C}, \boldsymbol{K}$ are generalized $n \times n$ mass, damping, and stiffness matrices of finite element model, $\mathbf{p}(\omega, t)$ is a vector of excitation forces, which, in our case, is periodic (unbalance force for rotating machines), and $\omega$ is the excitation frequency. The rotation effects, such as gyroscopic moments, Coriolis forces, centrifuge effect, etc, can be taken into account in the damping and stiffness term. Harmonic balance method (HBM) is utilized for its efficiency with respect to time domain method. The time variation of displacements and external forces for the steady-state periodic regime are represented by restricted Fourier series:

$$
\begin{aligned}
& \mathbf{x}(t)=\mathbf{X}^{0}+\sum_{k=0}^{N} \mathbf{X}_{c}^{k} \cos (k \omega t)+\mathbf{X}_{s}^{k} \sin (k \omega t) \\
& \mathbf{p}(t)=\mathbf{P}^{0}+\sum_{k=0}^{N} \mathbf{P}_{c}^{k} \cos (k \omega t)+\mathbf{P}_{s}^{k} \sin (k \omega t)
\end{aligned}
$$

where $\quad \mathbf{X}=\left[\mathbf{X}^{0}, \mathbf{X}_{c}{ }^{1}, \mathbf{X}_{s}{ }^{1}, . . \mathbf{X}_{c}{ }^{N}, \mathbf{X}_{s}{ }^{N}\right]^{T}, \quad \mathbf{P}=$ $\left[\mathbf{P}^{0}, \mathbf{P}_{c}^{1}, \mathbf{P}_{s}^{1}, . . \mathbf{P}_{c}^{N}, \mathbf{P}_{s}^{N}\right]^{T}$ are the vectors of Fourier coefficients for displacements and external excitation with truncated decomposition up to order $N$.

The main idea of harmonic balance method is to identify the periodic solution through estimations of the truncated Fourier coefficients by solving the nonlinear equation of motion in frequency domain. By substituting (2) (3) into (1), then applying a Galerkin procedure, the nonlinear differential equation (1) is transformed into a nonlinear algebraic equation system of dimension $n \times(2 N+1)$ :

$$
\mathbf{R}(\mathbf{X}, \omega, \lambda)=\mathbf{Z}(\omega) \mathbf{X}+\mathbf{F}_{N L}\left(\mathbf{X}, \lambda_{F}\right)-\mathbf{P}\left(\omega, \lambda_{p}\right)=0
$$

where $\mathbf{Z}=\operatorname{diag}\left(\mathbf{K}, \mathbf{Z}_{1}, . . \mathbf{Z}_{k}, . . \mathbf{Z}_{N}\right)$ with $\mathbf{Z}_{k}=\left[\begin{array}{cc}\mathbf{K}-k^{2} \omega^{2} \mathbf{M} & \omega \mathbf{C} \\ -\omega \mathbf{C} & \mathbf{K}-k^{2} \omega^{2} \mathbf{M}\end{array}\right], \mathbf{F}_{N L}$ and $\mathbf{P}$ are vectors of harmonic coefficients for nonlinear forces and excitation forces. $\lambda_{F}$ and $\lambda_{p}$ are parameters of nonlinear and excitation forces that can be varied later for parametric analysis.

A robust scheme, which obtains a set of discrete points to trace the equilibrium path in the range of parameters characterizing the structure, is based on Newton's method. The utilized Newton-Raphson method consists in an incrementaliterative procedure for approaching the exact solution

$$
\mathbf{J} \delta \mathbf{X}^{k}=-\mathbf{R}\left(\mathbf{X}^{k}\right)
$$

$$
\mathbf{X}^{k+1}=\mathbf{X}^{k}+\delta \mathbf{X}^{k}
$$

where $\mathbf{J}(\mathbf{X}, \omega, \lambda)=\frac{\partial \mathbf{R}^{k}}{\partial \mathbf{X}}=\mathbf{Z}(\omega)+\frac{\partial \mathbf{F}_{N L}}{\partial \mathbf{X}}$ is the Jacobian matrix, $\mathbf{R}\left(\mathbf{X}^{k}\right)$ the residue of the equilibrium equation of motion at iteration $k$. 
The Fourier coefficients of nonlinear efforts $\mathbf{F}_{N L}$ and its derivative $\frac{\partial \mathbf{F}_{N L}}{\partial \mathbf{X}}$ involved in the Newton-Raphson iterations are obtained by Alternating Frequency-Time (AFT) method [21]. The AFT scheme uses fast direct and inverse Fourier transforms to compute the nonlinear forces in the time domain and then switch back to the frequency domain for the reason that nonlinear forces are usually much easier to evaluate in the time domain than in the frequency domain.

Nonlinear systems often have several possible responses for a given excitation frequency $\omega$. The pseudo-arc length continuation method [22] combined with the abovementioned algorithm permits to follow the solution branch beyond turning points in order to obtain both stable and unstable solutions of response curves. The prediction step of continuation is firstly performed in the tangent direction to the solution curve. Then, this estimation is corrected iteratively in the orthogonal direction for the purpose of cancelling the residue $\mathbf{R}_{1}$ :

$\mathbf{R}_{1}(\mathbf{X}, \omega)=\mathbf{J}_{c}\left\{\begin{array}{l}\delta \mathbf{X}^{k+1} \\ \delta \omega^{k+1}\end{array}\right\}=\left[\begin{array}{cc}\mathbf{J} & \frac{\partial \mathbf{R}^{k}}{\partial \omega} \\ \Delta \mathbf{X}_{i}^{T} & \Delta \omega_{i}\end{array}\right]\left\{\begin{array}{l}\delta \mathbf{X}^{k+1} \\ \delta \omega^{k+1}\end{array}\right\}=\left\{\begin{array}{c}-\mathbf{R}^{k} \\ 0\end{array}\right\}$

where $\left[\Delta \mathbf{X}_{i}^{T} \Delta \omega_{i}\right]$ and $\left\{\delta \mathbf{X}^{k+1} \delta \omega^{k+1}\right\}^{t}$ stands for the unitary tangent vector and the orthogonal correction vector respectively, $k$ and $k+1$ correspond to the iteration number. The pseudo-arc length continuation method thus adds an equation and an unknown to the solving system. The iterative calculation is accomplished when the required accuracy is achieved. Consequently, the equilibrium path on the study range is obtained.

\section{LOCATION OF SINGULAR POINTS AND STA- BILITY ASSESSMENT}

Singular points that often accompanied by a change of stability, are generally distinguished into turning (limit) points and bifurcation points. In some cases, turning points are located near the vibration resonance. Besides, the analysis of bifurcation points has large practical importance since any of the multitude of branching solutions can be realized for the same operating conditions.

The determinant values of Jacobians $\mathbf{J}$ and $\mathbf{J}_{c}$ provide the information to classify the found solutions as follow:

$$
\begin{aligned}
& \text { regular point if } \operatorname{det}(\mathbf{J}) \neq 0 \text { and } \frac{\partial \mathbf{R}^{T}}{\partial \omega} \mathbf{y} \neq 0 \\
& \text { turning point if } \operatorname{det}(\mathbf{J})=0 \text { and } \frac{\partial \mathbf{R}^{T}}{\partial \omega} \mathbf{y} \neq 0 \\
& \text { bifurcation point if } \operatorname{det}(\mathbf{J})=0 \text { and } \frac{\partial \mathbf{R}^{T}}{\partial \omega} \mathbf{y}=0
\end{aligned}
$$

where $\mathbf{y}$ is the eigenvector associated with the zero eigenvalue of $\mathbf{J}$. During the continuation of the response curve, a sign change of $\operatorname{det}\left(\mathbf{J}_{c}\right)$ between two consecutive points indicates the presence of a bifurcation point.

To locate singular points precisely, it is more efficient to characterize the singular points using the equation $\mathbf{J y}=0$ rather than the determinant of $\mathbf{J}$. Between the two consecutive points, the one which has a smaller absolute value of $\operatorname{det}(\mathbf{J})$ is chosen to approach the exact singular point. which leads to solve the following augmented system for each iteration of Newton-Raphson:

$$
\left[\begin{array}{ccc}
\mathbf{J} & 0 & \frac{\partial \mathbf{R}}{\partial \omega} \\
\frac{\partial(\mathbf{J y})}{\partial X} & \mathbf{J} & \frac{\partial(\mathbf{J y})}{\partial \omega} \\
0 & 2 \mathbf{y}^{T} & 0
\end{array}\right]\left\{\begin{array}{c}
\delta \mathbf{X} \\
\delta \mathbf{y} \\
\delta \omega
\end{array}\right\}=-\left\{\begin{array}{c}
\mathbf{R}+\gamma \mathbf{e}_{j} \\
\mathbf{J y} \\
\|\mathbf{y}\|^{2}-1
\end{array}\right\}
$$

$$
\begin{array}{rlrl}
\mathbf{J}=\mathbf{Z}+\frac{\partial \mathbf{F}_{N L}}{\partial \mathbf{X}} & \frac{\partial(\mathbf{J} \mathbf{y})}{\partial \mathbf{X}}=\frac{\partial^{2} \mathbf{F}_{N L}}{\partial \mathbf{X}^{2}} \mathbf{y} \\
\text { with } & \frac{\partial \mathbf{R}}{\partial \omega}=\frac{\partial \mathbf{Z}}{\partial \omega} \mathbf{X}-\frac{\partial \mathbf{P}}{\partial w} & \frac{\partial(\mathbf{J y})}{\partial \omega}=\frac{\partial \mathbf{Z}}{\partial \omega} \mathbf{y}
\end{array}
$$

For locating a bifurcation points, an additional equation $\frac{\partial \mathbf{R}^{T}}{\partial \omega} \mathbf{y}=0$ and an new unknown $\gamma$ are added. The term $\gamma \mathbf{e}_{j}$ in (9) is added only when locating a bifurcation point where $\mathbf{e}_{j}$ is a unit vector with $j$-th component equal to $1, \gamma$ is an auxiliary variable for eliminating the singularity of Jacobian [17] and its initial value is assumed to be 0 . The approximation of the eigenvector $\mathbf{y}$ is calculated by performing singular value decomposition of the Jacobian and selecting a vector corresponding to the minimum magnitude of the singular value.

This algorithm applies only to simple singular points. In more complex cases, such as the change of dynamic regime form periodic to quasi-periodic (see §5.2), the detection of the bifurcation point can be performed by means of a classical stability assessment.

Assessment of the local stability consists in applying a small perturbation to the equilibrium solution, and then checking whether or not this perturbation subsides with time. Floquet theory is the most widely used method for evaluating the stability of solution. There are several algorithms, both in the time and frequency domain, to compute the eigenvalues of the so-called monodromy matrix [20].

In this work, the monodromy matrix $\Phi=[\phi \dot{\phi}]$ is obtained by integrating the following system:

$$
\mathbf{M} \ddot{\phi}(t)+\left(\mathbf{C}+\frac{\partial \mathbf{f}_{n l}}{\partial \dot{x}}\right) \dot{\phi}(t)+\left(\mathbf{K}+\frac{\partial \mathbf{f}_{n l}}{\partial x}\right) \phi(t)=\frac{\partial \mathbf{p}}{\partial x}
$$

over one period of motion with the Newmark algorithm.

The eigenvalues of the monodromy matrix, also known as the Floquet multipliers, provide information on the stability of the periodic solution. If all the multipliers are inside the unit circle in the complex plane $\left(R_{e}, I_{m}\right)$, the corresponding solution is asymptotically stable and called a stable limit cycle. If at least one multiplier is outside the unit circle, there is a local bifurcation of limit cycle with a loss of stability.

\section{PARAMETRIC CONTINUATION OF SINGULAR POINTS - STABILITY BOUNDARY DETERMINA- TION}

Once the singular point $\left(\mathbf{X}_{0}, \omega_{0}\right)$ is specifically detected along the equilibrium branch, it is possible to follow its vari- 
ation with respect to a parameter $\lambda\left(\lambda_{F}, \lambda_{p}\right)$ with a continuation method similar to that described in paragraph $\S 3$. In order to do this, $\lambda$ is considered as a new unknown and an equation $r_{2}(\mathbf{X}, \omega, \lambda)=0$ is added to the augmented system (9), which requires the Newton-Raphson corrections to be orthogonal to the prediction step. The continuation of the singular point is thus conducted by successive corrections in the following form:

$$
\left[\begin{array}{ccccc}
\mathbf{J} & \mathbf{0} & \frac{\partial \mathbf{R}}{\partial \omega} & \mathbf{e}_{j} & \frac{\partial \mathbf{R}}{\partial \lambda} \\
\frac{\partial(\mathbf{J y})}{\partial X} & \mathbf{J} & \frac{\partial(\mathbf{J} \mathbf{y})}{\partial \omega} & \mathbf{0} & \frac{\partial(\mathbf{J y})}{\partial \lambda} \\
\mathbf{0} & 2 \mathbf{y}^{T} & 0 & 0 & 0 \\
\mathbf{0} & \frac{\partial \mathbf{R}^{T}}{\partial \omega} & \mathbf{0} & \mathbf{0} & 0 \\
\Delta \mathbf{X}^{T} & \mathbf{0} & \Delta \omega & 0 & \Delta \lambda
\end{array}\right]\left\{\begin{array}{c}
\delta \mathbf{X} \\
\delta \mathbf{y} \\
\delta \omega \\
\delta \gamma \\
\delta \lambda
\end{array}\right\}=-\left\{\begin{array}{c}
\mathbf{R}+\gamma \mathbf{e}_{j} \\
\mathbf{J y} \\
\|\mathbf{y}\|^{2}-1 \\
\frac{\partial \mathbf{R}^{T}}{\partial \omega} \mathbf{y} \\
r_{2}
\end{array}\right\}
$$

with

$$
\frac{\partial \mathbf{R}}{\partial \lambda}=\frac{\partial \mathbf{F}_{N L}}{\partial \lambda}-\frac{\partial \mathbf{P}}{\partial \lambda} \quad \frac{\partial(\mathbf{J y})}{\partial \lambda}=\frac{\partial^{2} \mathbf{F}_{N L}}{\partial \mathbf{X} \partial \lambda} \mathbf{y}
$$

Again, the term $\gamma \mathbf{e}_{j}$ and $\delta \gamma$ are necessary to eliminate the singularity of the augmented system during the bifurcation point continuation.

\section{NUMERICAL RESULTS}

Numerical simulations are presented in this section in order to demonstrate the validity and the advantage of the proposed approach.

\subsection{Duffing oscillator}

The Duffing oscillator is a one-degree of freedom system with a nonlinear restoring force proportional to the cube of the displacement. The accuracy of the proposed method in predicting the periodic motions of the forced Duffing oscillator is examined by comparing the stable branch obtained by HBM (19 harmonics) with those obtained from direct temporal integration. The system is described by the differential equation:

$$
m \ddot{x}(t)+c \dot{x}+k x(t)+\alpha x(t)^{3}=p_{0} \cos (\omega t)
$$

where $\alpha$ is the nonlinear stiffness coefficient, and $p_{0}$ is the excitation amplitude.

Using the numerical continuation of periodic solutions with the excitation frequency as the continuation parameter, the assessment is firstly carried out with the parameters: $m=$ $1, c=0.1, k=1, \alpha=1, p_{0}=0.5$. The frequency response curve is shown in Fig.1. A classical resonance curve can be observed while the blue dots and red dots stand for stable and unstable solutions respectively. The two magenta points are obtained by monitoring the sign change of the conditions in (8). Since only $\operatorname{det}(\mathbf{J})$ passed through 0 , these are limit points with stability change and no bifurcation occurs.

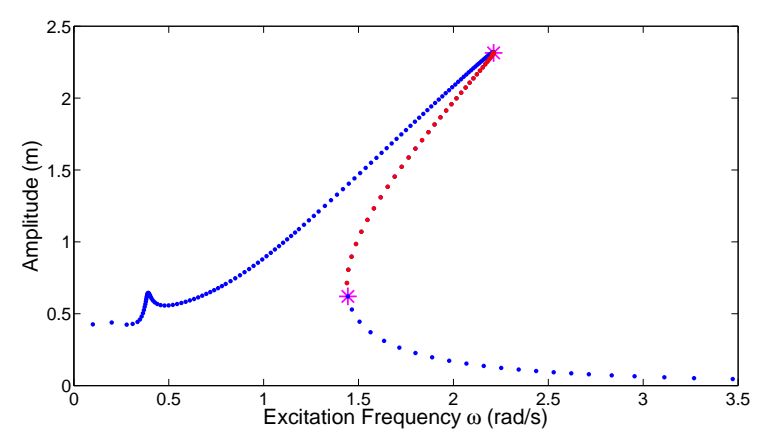

Fig. 1. Frequency-response curve of the Duffing system for $m=1$, $c=0.1, k=1, \alpha=1, p_{0}=0.5$. (blue dot : stable solution; red dot : unstable solution; magenta point : turning point)

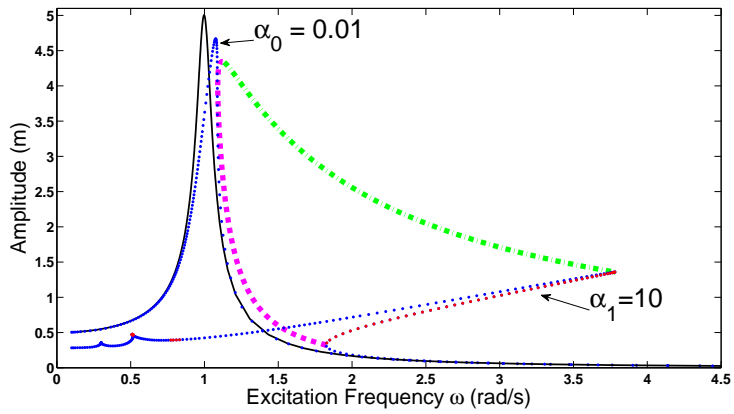

Fig. 2. Turning point tracking: parametric variation of stability limits of the Duffing oscillator in function of $\alpha \in[0.0110]$ with $m=1$, $c=0.1, k=1, p_{0}=0.5$. (black line : linear response; blue dot : stable solution; red dot : unstable solution; green and magenta lines : turning point tracking

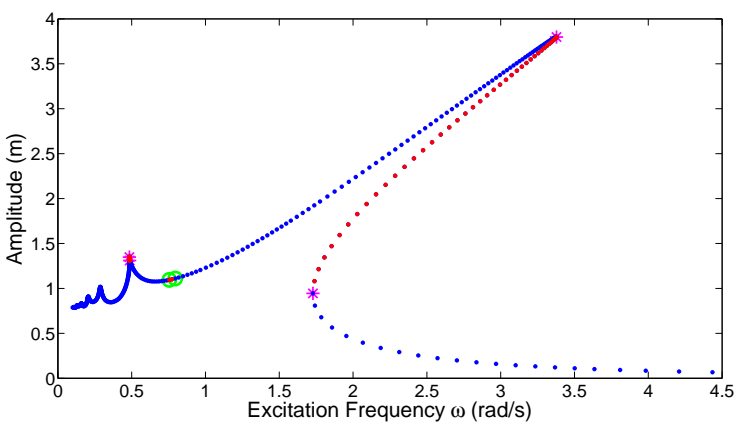

Fig. 3. Frequency-response curve of the Duffing system for $m=1$, $c=0.1, k=1, \alpha=1, p_{0}=1.25$. (blue dot : stable solution; red dot : unstable solution; magenta point: turning point; green circle: saddle-node bifurcation)

By applying the continuation technique presented above, the two limit points are followed when the nonlinear coefficient $\alpha$ is varied from 0.01 to 10. In Fig.2, the response curve of the linear system $(\alpha=0)$ is plotted in black line, the blue dotted curve nearby is the system response for $\alpha_{0}=0.01$ (slightly nonlinear) which is totally stable, while the response on the right is calculated for $\alpha_{1}=10$ (strongly 


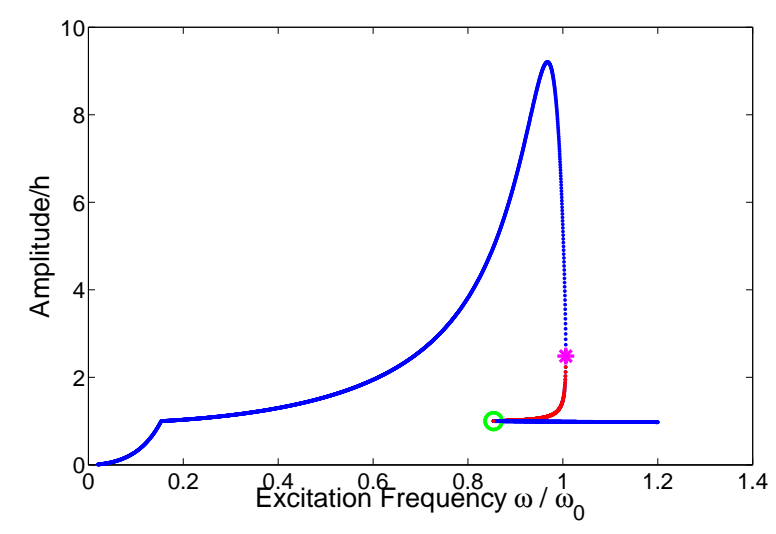

Fig. 4. Forced response curve of Jeffcott rotor for $\mu=0$. (blue dot : stable solution; red dot : unstable solution; magenta point : turning point; green circle: saddle-node bifurcation)

nonlinear). The green dotted-line represents the direct tracking of the first limit point while the magenta dotted-line tracks the lower limit point. The zone that between these two dotted-lines is the unstable area of the Duffing system when its nonlinear coefficient varies. The two lines are the stability boundaries defining amplitude jump: when frequency increases, am amplitude jump arises when the system vibration level comes close to the green line, and when frequency decreases, an amplitude jump will be predicted by the red line.

When the nonlinear effect is stronger, or the excitation force is higher, bifurcation points are found as shown by green circles in Fig.3. Besides of the two limit points previously studied, there are other limit points at the superharmonic resonances.

\subsection{Nonlinear Jeffcott rotor}

The second test case is a modified Jeffcott rotor which can come into contact with a stator that is modeled as an added stiffness [1] [3]. The rotor is made of a weightless shaft carrying a disk with mass $m$ at the middle of the span. The clearance between the rotor and the stator is denoted by $h$. The stator, which is rigidly fixed, has an elastic contact surface modeled as a symmetrical set of radial springs with isotropic stiffness, $k_{c}$. The equations of motion are shown below

$$
\begin{aligned}
& m \ddot{x}+c \dot{x}+k x+k_{c}\left(1-\frac{h}{r}\right)\left(x-\mu y \operatorname{sign}\left(v_{r e l}\right)\right)=p_{b} \omega^{2} \cos \omega t \\
& m \ddot{y}+c \dot{y}+k y+k_{c}\left(1-\frac{h}{r}\right)\left(\mu x \operatorname{sign}\left(v_{r e l}\right)+y\right)=p_{b} \omega^{2} \cos \omega t
\end{aligned}
$$

where $k$ is the stiffness of the shaft, $r=\sqrt{x^{2}+y^{2}}$ is the radial displacement, $p_{b}$ is the imbalance amplitude and $v_{r e l}$ is the relative velocity between the rotor and the stator at the contact point.

All the calculations are carried out with the same conditions: $m=1, c=5, k=100, k_{c}=2500, h=0.105, p_{b}=0.1$, except that the friction coefficient $\mu$ is considered as the var-

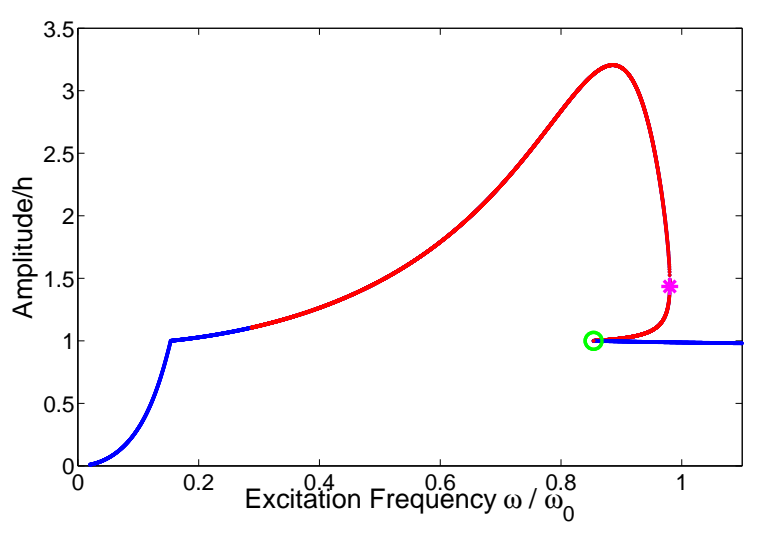

Fig. 5. Forced response curve of Jeffcott rotor for $\mu=0.2$. (blue dot : stable solution; red dot : unstable solution; magenta point : turning point; green circle: saddle-node bifurcation)

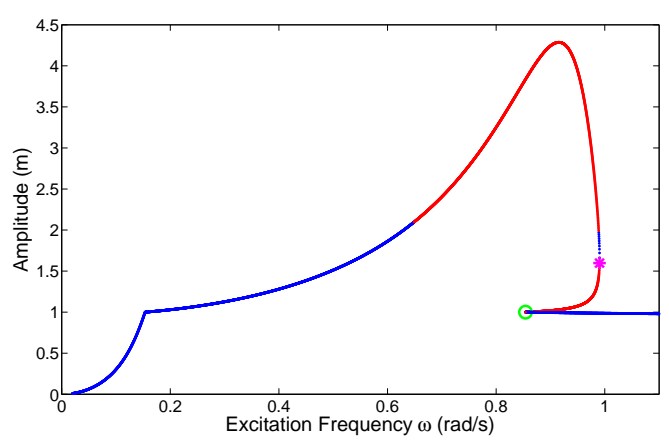

Fig. 6. Forced response curve of Jeffcott rotor for $\mu=0.12$. (blue dot : stable solution; red dot : unstable solution; magenta point : turning point; green circle: saddle-node bifurcation)

ied parameter. Firstly, the response curve of the system when $\mu=0$ is plotted in Fig.4. Both the detection of sign change of Jacobian determinant and the stability assessment were performed. As shown, the red dots indicate the unstable branch while the magenta points mark the limit points. The response has the same shape for $\mu=0.1$.

Yet, when the friction coefficient increases to 0.12 then to 0.2 , the stability distribution changes a lot. In Fig.5, the singular points are marked, however, the stability change next to the dimensionless frequency 0.3 doesn't cause a sign change in the Jacobian determinant. In fact, it's related to the regime change of the system from synchronous full annular rub motion to partial rub motion, which means the stable periodic motion changes to quasi-periodic motion [23]. This fact indicates that monitoring the sign change of Jacobian is efficient to provide information on simple singular points only (saddle-node, asymmetric, and pitchfork bifurcations), but it is insufficient to predict all the stability change of system solutions. Constraint equations and associated extended systems for tracking complex bifurcations (period doubling, Neimark-Sacker) can be found in [24]. Their use in the framework of the HBM are beyond the scope of this paper. Figure. 6 shows another stable branch between the two unsta- 


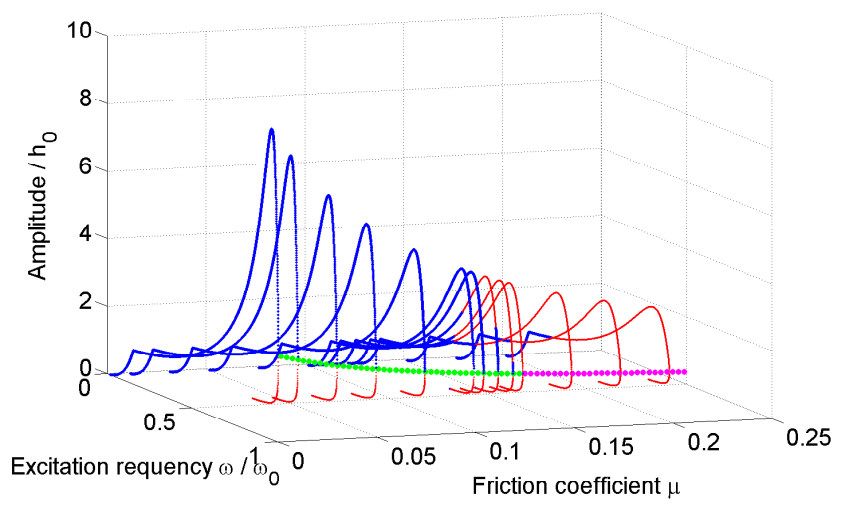

Fig. 7. Singular point tracking of Jeffcott rotor as a function of friction coefficient $\mu$. (blue branch : stable solution; red branch : unstable solution; green point : stable solution; magenta point : unstable solution

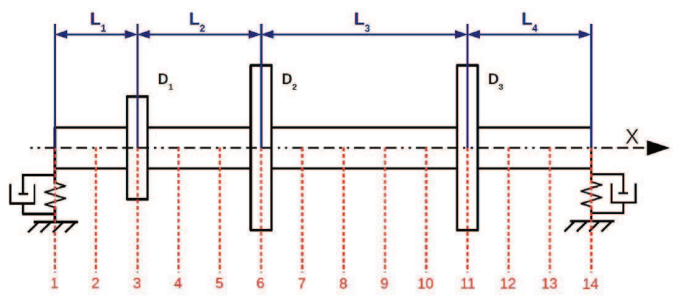

Fig. 8. Multi-DOFs finite element rotor [20]

ble branches indicating that partial rub motion changes back to synchronous full annular rub.

Figure.7 collects all the calculated responses for several values of friction coefficient $\mu$. Blue and red lines indicate stable and unstable branches respectively. The half-green half-magenta line is calculated with the continuation technique which permets a direct following of the bifurcation points with respect to $\mu$. The stability analysis of converged solution on this line distinguishes stable solutions (green) from unstable ones (magenta). As observed, the continuation technique succeeds to locate directly all the bifurcation points. Where they correspond to a change of stability in the response curve, they are stable (green branch), whereas they are unstable (magenta curve) when there is no stability change involved.

\subsection{Finite element nonlinear rotor}

A finite element nonlinear rotor model [25] [20] was also tested (see Fig.8). This rotor is composed of 13 Timoshenko beam elements. Two linear isotropic mountings are located at both ends of the rotor $\left(k_{y y}=k_{z z}=6 e 7 \mathrm{~N} . \mathrm{m}^{-3}\right.$, $c_{y y}=c_{z z}=600$ N.s.m), and three disks (modeled by additional mass and inertia) are added to nodes 3,6 and 11. The aim is to calculate its response with an imbalance force located on disk $2\left(m_{b} . g=2 e-2 \mathrm{~kg} . \mathrm{m}\right)$. The rotor makes contact with a circular, static, and rigid stator located in the vicinity of disk 2 . The contact model is identical to that de-

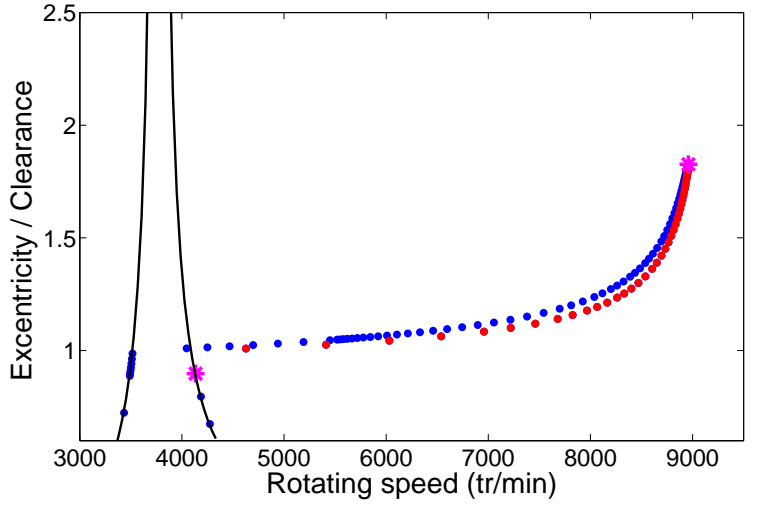

Fig. 9. Forced response curve of finit element rotor for $\mu=0$ (blue dot : stable solution; red dot : unstable solution; magenta point : turning point)

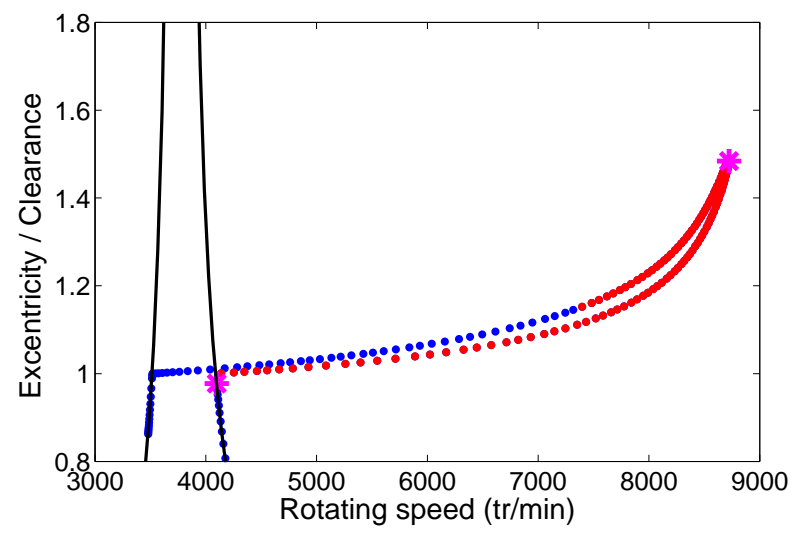

Fig. 10. Forced response curve of finite element rotor for $\mu=0.03$ (blue dot : stable solution; red dot : unstable solution; magenta point : turning point)

scribed in section $\S 5.2$ with the initial clearance $h=1 \mathrm{~mm}$. The equation of motion is written in a finite element form:

$$
\mathbf{M} \ddot{\mathbf{x}}(t)+\mathbf{C}(\omega) \dot{\mathbf{x}}(t)+\mathbf{K x}(t)+\mathbf{f}_{c}(\mathbf{x})=\mathbf{p}(\omega, t)
$$

where $\mathbf{M}, \mathbf{C}(\omega)$ and $\mathbf{K}$ represent the mass, damping and stiffness matrices respectively. $\mathbf{C}(\omega)$ includes the gyroscopic matrix, which varies with $\omega$.

The calculations for finite element rotor are carried out in Cast $3 \mathrm{~m}$ [26]. Figure. 9 represents the response curve with stable and unstable branches for the friction coefficient $\mu=0$. The black line stands for linear response. Two turning points are also detected. The response curve for $\mu=0.03$ is shown in Fig.10. Same as in Fig.5, the system has passed stable and unstable phases due to complex motion changes due to the strong non-linearity arising from contact. Similarly, not all bifurcations have been detected.

In Fig. 11 the magenta line is directly calculated with the continuation technique and covers all the bifurcation points with respect to $\mu$ (from 0 to 0.2 ). This example illustrates 


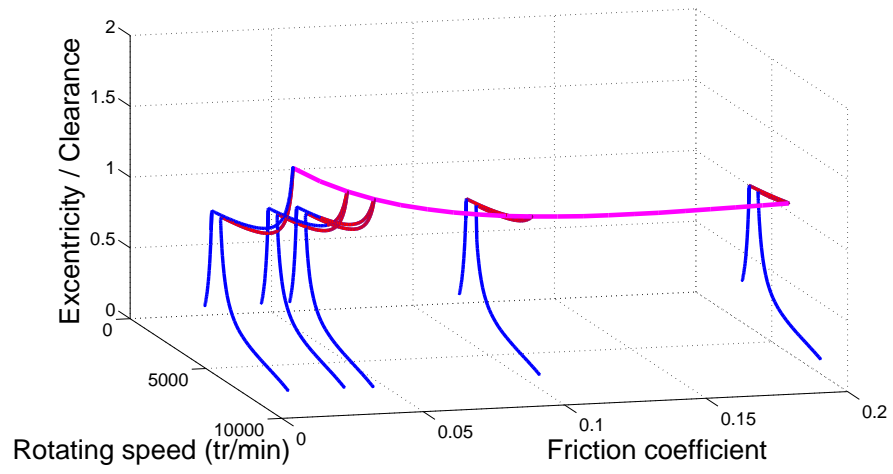

Fig. 11. Singular point tracking of finite element rotor as a function of friction coefficient $\mu$. (blue branch : stable solution; red branch : unstable solution; magenta point : singular points )

that the continuation technique is able to locate directly the bifurcation points in applications of finite element analysis.

\section{CONCLUSIONS}

An efficient frequency-domain method is presented for the rapid parametric analysis of stability changes in nonlinear dynamic systems, which provides directly the stability boundary with respect to parameters such as the system nonlinearity or excitation level.

First, the equilibrium solutions are calculated by continuation technique combined with harmonic balance method. Then stability of equilibrium solutions is determined by Floquet theory. The determinant of Jacobian provides information on limit points and bifurcation points. After locating these singular points, the proposed method is able to follow them directly with respect to a varied parameter. The application examples have illustrated the efficiency and accuracy of proposed method.

Numerical developments are fulfilled in both Matlab and Cast $3 \mathrm{~m}$, paving the way for application of the method to the nonlinear dynamics of rotors modelized with 3D finite elements.

\section{References}

[1] Groll, G. V., and Ewins, D., 2001. "The harmonic balance method with arc-length continuation in rotor/stator contact problems". Journal of Sound and Vibration, 241(2), pp. 223 - 233.

[2] Petrov, E., and Ewins, D., 2004. "State-of-the-art dynamic analysis for non-linear gas turbine structures". Proceedings of the Institution of Mechanical Engineers, Part G: Journal of Aerospace Engineering, 218(3), pp. 199-211.

[3] Peletan, L., Baguet, S., Torkhani, M., and JacquetRichardet, G., 2014. "Quasi-periodic harmonic balance method for rubbing self-induced vibrations in rotor tator dynamics". Nonlinear Dynamics, 78(4), pp. 2501-2515.
[4] Chen, C., Dai, L., and Fu, Y., 2007. "Nonlinear response and dynamic stability of a cracked rotor". Communications in Nonlinear Science and Numerical Simulation, 12(6), pp. $1023-1037$.

[5] Cheng, L., Li, N., Chen, X., and He, Z., 2011. "The influence of crack breathing and imbalance orientation angle on the characteristics of the critical speed of a cracked rotor". Journal of Sound and Vibration, 330(9), pp. 2031 - 2048.

[6] Khonsari, M. M., and Chang, Y. J., 1993. "Stability boundary of non-linear orbits within clearance circle of journal bearings". Journal of Vibration and Acoustics, 115, pp. 303-307.

[7] Brown, R., Addison, P., and Chan, A., 1994. "Chaos in the unbalance response of journal bearings". Nonlinear Dynamics, 5(4), pp. 421-432.

[8] Dakel, M. Z., Baguet, S., and Dufour, R., 2014. "Nonlinear dynamics of a support-excited flexible rotor with hydrodynamic journal bearings". Journal of Sound and Vibration, 333(10), pp. 2774-2799.

[9] Petrov, E. P., 2007. "Direct parametric analysis of resonance regimes for nonlinear vibrations of bladed disks”. J. TurboMach., 129(3), pp. 495-502.

[10] Petrov, E. P., 2009. "Method for sensitivity analysis of resonance forced response of bladed disks with nonlinear contact interfaces". Journal of Engineering for Gas Turbines and Power, 131 (2).

[11] Liao, H., and Wang, J., 2013. "Maximization of the vibration amplitude and bifurcation analysis of nonlinear systems using the constrained optimization shooting method". Journal of Sound and Vibration, 332(16), pp. 3781-3793.

[12] Balaram, B., Narayanan, M., and Rajendrakumar, P., 2012. "Optimal design of multi-parametric nonlinear systems using a parametric continuation based genetic algorithm approach". Nonlinear Dynamics, 67(4), pp. 2759-2777.

[13] Nayfeh, A. H., and Mook, D. T., 1995. Nonlinear oscillations. Wiley New York.

[14] Moore, G., and Spence, A., 1980. "The calculation of turning points of nonlinear equations". SIAM Journal on Numerical Analysis, 17(4), pp. 567-576.

[15] Wriggers, P., and Simo, J. C., 1990. "A general procedure for the direct computation of turning and bifurcation points". Int. J. Numer. Meth. Engng., 30.

[16] Battini, J.-M., Pacoste, C., and Eriksson, A., 2003. "Improved minimal augmentation procedure for the direct computation of critical points". Computer Methods in Applied Mechanics and Engineering, 192(16â18), pp. 2169-2185.

[17] Felippa, C. A., 1987. "Traversing critical points by penalty springs". Proceedings of NUMETA'87 Conference, Swansea, Wales, Nijhoff Pubs, Dordrecht, Holland, .

[18] Liaw, C., and Koh, C., 1993. "Dynamic stability and chaos of system with piecewise linear stiffness". Journal of Engineering Mechanics, 119(8), pp. 1542-1557.

[19] Kim, Y.-B., and Noah, S., 1996. "Quasi-periodic re- 
sponse and stability analysis for a non-linear jeffcott rotor". Journal of Sound and Vibration, 190(2), pp. 239 $-253$.

[20] Peletan, L., Baguet, S., Torkhani, M., and JacquetRichardet, G., 2013. "A comparison of stability computational methods for periodic solution of nonlinear problems with application to rotordynamics". Nonlinear Dynamics, 72(3), pp. 671-682.

[21] Cameron, T. M., and Griffin, J. H., 1989. "An alternating frequency/time domain method for calculating the steady-state response of nonlinear dynamic systems". Journal of Applied Mechanics, 56, pp. 149-154.

[22] Crisfield, M., 1981. "A fast incremental/iterative solution procedure that handles snap-through". Computers \& Structures, 13(1â3), pp. 55-62.

[23] Jiang, J., 2009. "Determination of the global responses characteristics ofapiecewise smooth dynamical system with contact". Nonlinear Dynamics, 57(3), pp. 351361.

[24] Doedel, E. J., Govaerts, W., and Kuznetsov, Y. A., 2003. "Computation of periodic solution bifurcations in odes using bordered systems". SIAM J. Numer. Anal., 41(2), Feb., pp. 401-435.

[25] Lalanne, M., and Ferraris, G., 1998. Rotordynamics prediction in engineering. No. vol. 2 in Rotordynamics prediction in engineering. John Wiley.

[26] CAST3M, 2014. "http://www-cast3m.cea.fr/". CEA (French Atomic Energy Commission). 